\title{
Low- versus Standard-Dose Intravenous Alteplase in the Context of Bridging Therapy for Acute Ischemic Stroke: A Korean ENCHANTED Study
}

\author{
Jong S. Kim, ${ }^{\text {a } Y e o n-J u n g ~ K i m, ~}{ }^{\mathrm{a}}$ Kyung Bok Lee, ${ }^{\mathrm{b}}$ Jae Kwan Cha, ${ }^{\mathrm{c}}$ Jong-Moo Park, ${ }^{\mathrm{d}}$ Yangha Hwang, \\ Eung-Gyu Kim, ${ }^{\mathrm{f}}$ Joung-Ho Rha, ${ }^{\mathrm{g}}$ Jaseong Koo, ${ }^{\mathrm{h}}$ Jei Kim, ${ }^{\mathrm{i}}$ Yong-Jae Kim, ${ }^{\mathrm{j}}$ Woo-Keun Seo, ${ }^{\mathrm{k}}$ \\ Dong-Eog Kim, ${ }^{\mathrm{l}}$ Thompson G. Robinson, ${ }^{\mathrm{m}}$ Richard I. Lindley, ${ }^{\mathrm{n}, \mathrm{o}}$ Xia Wang, ${ }^{\mathrm{o}, \mathrm{p}}$ John Chalmers, ${ }^{\mathrm{o}, \mathrm{p}}$ \\ Craig S. Anderson ${ }^{\mathrm{o}, \mathrm{p}, \mathrm{q}, \mathrm{r}}$ \\ aDepartment of Neurology, Asan Medical Center, University of Ulsan College of Medicine, Seoul, Korea \\ bDepartment of Neurology, Soonchunhyang University Seoul Hospital, Soonchunhyang University College of Medicine, Seoul, Korea \\ 'Department of Neurology, Dong-A University Medical Center, Dong-A University College of Medicine, Busan, Korea \\ ${ }^{d}$ Department of Neurology, Eulji General Hospital, Eulji University School of Medicine, Seoul, Korea \\ eDepartment of Neurology, Kyungpook National University Hospital, Kyungpook National University School of Medicine, Daegu, Korea \\ fDepartment of Neurology, Inje University Busan Paik Hospital, Inje University College of Medicine, Busan, Korea \\ ${ }^{9}$ Department of Neurology, Inha University Hospital, Inha University School of Medicine, Incheon, Korea \\ hDepartment of Neurology, Seoul St. Mary's Hospital, College of Medicine, The Catholic University of Korea, Seoul, Korea \\ 'Department of Neurology, Chungnam National University School of Medicine, Daejeon, Korea \\ 'Department of Neurology, Ewha Womans University Mokdong Hospital, Ewha Womans University School of Medicine, Seoul, Korea \\ kDepartment of Neurology, Samsung Medical Center, Sungkyunkwan University School of Medicine, Seoul Korea \\ 'Department of Neurology, Dongguk University Ilsan Hospital, Dongguk University College of Medicine, Goyang, Korea \\ ${ }^{m}$ Department of Cardiovascular Sciences and NIHR Biomedical Research Unit, University of Leicester, Leicester, UK \\ "Westmead Clinical School, University of Sydney, Westmead, Australia \\ 'Sydney Medical School, University of Sydney, Sydney, Australia \\ PThe George Institute for Global Health, University of New South Wales, Newtown, Australia \\ "Department of Neurology, Royal Prince Alfred Hospital, Sydney Health Partners, Sydney, Australia \\ 'The George Institute China at Peking University Health Science Center, Beijing, China
}

Background and Purpose Following the positive results from recent trials on endovascular therapy (EVT), bridging therapy (intravenous alteplase plus EVT) is increasingly being used for the treatment of acute ischemic stroke. However, the optimal dose of intravenous alteplase remains unknown in centers where bridging therapy is actively performed. The optimal dose for eventual recanalization and positive clinical outcomes in patients receiving bridging therapy also remains unknown.

Methods In this prospective Enhanced Control of Hypertension and Thrombolysis Stroke Study (ENCHANTED) sub-study, we explored the outcomes following treatment with two different doses (low- $[0.6 \mathrm{mg} / \mathrm{kg}]$ or standard-dose $[0.9 \mathrm{mg} / \mathrm{kg}]$ ) of intravenous alteplase across 12 Korean centers where EVT is actively performed. The primary endpoint was a favorable outcome at 90 days (modified Rankin Scale scores 0 to 1). Secondary endpoints included symptomatic intracerebral hemorrhage $(\mathrm{ICH})$ in all patients, and the recanalization rate and favorable outcome in patients who underwent cerebral angiography for EVT (ClinicalTrials.gov, number NCT01422616).

Results Of 351 patients, the primary outcome occurred in 46\% of patients in both the standard(80/173) and low-dose (81/178) groups (odds ratio [OR], 1.14; 95\% confidence interval [CI], 0.72 to
Correspondence: Jong S. Kim Department of Neurology, Asan Medical Center, University of Ulsan College of Medicine, 88 Olympic-ro, 43-gil, Songpa-gu, Seoul 05505, Korea Tel: $+82-2-3010-3442$ Fax: +82-2-474-4691 E-mail: jongskim@amc.seoul.kr

Co-correspondence: Craig S. Anderson The George Institute for Global Health, University of New South Wales, PO Box M201, Missenden Road, Newtown, New South Wales 2050, Australia

Tel: +86-15210347573

Fax: +86-1082800177

E-mail: canderson@georgeinstitute.org.au

Received: July 17, 2017

Revised: November 15, 2017

Accepted: November 22, 2017 
1.81; $P=0.582$ ), although ICHs tended to occur more frequently in the standard-dose group (8\% vs. $3 \%, P=0.056$ ). Of the 67 patients who underwent cerebral angiography, there was no significant difference in favorable functional outcome between the standard- and low-dose groups (39\% vs. $21 \% ; \mathrm{OR}, 2.39 ; 95 \% \mathrm{Cl}, 0.73$ to $7.78 ; P=0.149$ ).

Conclusions There was no difference in functional outcome between the patients receiving different doses of alteplase in centers actively performing bridging therapy.

Keywords Cerebral infarction; Thrombectomy; Intracranial hemorrhages

\section{Introduction}

The Enhanced Control of Hypertension and Thrombolysis Stroke Study (ENCHANTED) evaluated the effectiveness of standardversus low-dose intravenous alteplase in 3,310 thrombolysiseligible acute ischemic stroke patients between 2012 and 2015. This trial did not find that low-dose alteplase was noninferior to standard-dose alteplase with respect to death and disability at 90 days, although there were fewer symptomatic intracerebral hemorrhages ( $\mathrm{sICHs}$ ) in patients receiving lowdose alteplase. Because endovascular therapy (EVT) was rarely performed across the trial network during the study period, the ENCHANTED results are primarily applicable to patients who only received intravenous therapy.

It thus remains unknown what the appropriate alteplase dose is when additional EVT is performed. Notably, among the 13 countries participating in ENCHANTED, South Korea is the only country where EVT in addition to intravenous therapy (bridging therapy) is actively performed as part of routine clinical management. We therefore prospectively analyzed the Korean data to investigate potential differences in outcomes according to the different doses of alteplase used in the context of active bridging therapy. This is particularly meaningful because just after ENCHANTED patients were recruited, large clinical trials established the benefit of reperfusion therapy with EVT for patients with acute ischemic stroke due to large artery occlusion. ${ }^{2-7}$ With the number of patients receiving bridging therapy increasing worldwide, it is important to establish an appropriate alteplase dose in the context of bridging therapy.

The aim of the present study was to compare the effectiveness of standard- versus low-dose intravenous alteplase in acute stroke patients in the health care setting of active bridging therapy. We also examined whether the proportion of patients who need EVT differs based on different alteplase dose. Additionally, we assessed eventual recanalization rate and functional outcome in patients who underwent bridging therapy. For this study, we prospectively collected data from South Korean centers involved in the ENCHANTED study.

\section{Methods}

\section{Study design}

The design of ENCHANTED has been described elsewhere., ${ }^{8,9}$ Briefly, this was an international, multi-center, prospective, randomized, open-label trial with blinded outcome evaluation (PROBE design) that compared low-dose alteplase $(0.6 \mathrm{mg} / \mathrm{kg}$; 15\% bolus, remainder by infusion over 1 hour) and standarddose alteplase $(0.9 \mathrm{mg} / \mathrm{kg} ; 10 \%$ bolus, remainder by infusion over 1 hour). All patients were adults ( $\geq 18$ years of age) seen within 4.5 hours of acute ischemic stroke onset who fulfilled standard criteria for use of thrombolysis treatment. ${ }^{1}$ Randomization was achieved via a central internet-based system developed by the George Institute (Sydney, Australia). To ensure balance in key prognostic factors, randomization was stratified by the site of recruitment, time since onset ( $<3$ hours vs. $\geq 3$ hours), and the National Institutes of Health Stroke Scale (NIHSS) score ( $<10$ vs. $\geq 10)$.

For the current study, an additional one-page data sheet that included information on the performance of cerebral angiography and EVT, location of occluded vessel(s), EVT procedures, degree of recanalization, and intracerebral hemorrhage (ICH) occurrence (see below for definition) were prospectively obtained from the 12 Korean centers (Asan Medical Center, Chungnam National University Hospital, Dong-A University Medical Center, Dongguk University Ilsan Hospital, Eulji General Hospital, Ewha Womans University Mokdong Hospital, Inha University Hospital, Inje University Busan Paik Hospital, Korea University Guro Hospital, Kyungpook National University Hospital, Seoul St. Mary's Hospital, and Soonchunhyang University Seoul Hospital).

In nine of these centers, computed tomography (CT) was the primary imaging modality used to decide if alteplase should be administered. In the other three centers, either CT or magnetic resonance imaging (MRI) was used for the initial assessment. In all of the centers, following intravenous alteplase administration, patients underwent MRI, including both diffusion weighted MRI (DWI) and perfusion weighted MRI (PWI), as 
well as MR angiography. If the MR angiography revealed a large cerebral artery occlusion, patients were moved to an angiogram room and transfemoral angiography was performed in an attempt to perform EVT. The detailed processes following intravenous alteplase therapy and the EVT procedures were subject to the individual hospital policies and subtle differences may have occurred based on various factors, such as the availability of interventionists, physician interpretation of DWI/PWI mismatch, and subjective decision of 'large artery occlusion' that should be treated by EVT, e.g., inclusion of M2 portion of the middle cerebral artery. Thus, aside from the strict randomization of alteplase dose, we allowed the individual treatment strategy to vary among different hospitals.

The study protocol was approved by the Institutional Review Boards of the participating centers. All patients or their legally authorized representatives gave written informed consent to participate in this study.

\section{Clinical assessments and outcomes}

Patients were assessed daily for 1 week and then at 28 and 90 days (unless death occurred earlier) by an examiner who was blind to the treatment allocation. Follow-up data were collected 24 and 72 hours as well as 7 (or at time of death or hospital discharge if sooner), 28, and 90 days following the randomization. The modified Rankin Scale (mRS) was used to assess functional outcome $^{10}$ and the NIHSS was used to quantify neurologic deficit." Brain imaging (CT or MRI) was conducted using standardized techniques at baseline, within 24 hours following the procedure, and at the time at which survivors deteriorated for any reason. $\mathrm{sICH}$ was defined using the National Institute of Neurological Disorders and Stroke (NINDS) criteria, ${ }_{1}^{12}$ which included ICH with deterioration of at least 1 point on the NIHSS score or death within 36 hours. Consistent with other trials ${ }^{13,14}$ we subcategorized sICH as 'major' ( $\geq 4$ points increase on the NIHSS or death) or 'minor' (1 to 3 points increase on the NIHSS) deterioration over 72 hours. Early neurological improvement was defined as a reduction of at least 8 points in the NIHSS score or a score of 0 or 1 three days following the randomization. ${ }^{5}$

The modified-thrombolysis in cerebral infarction (TICI) score, ${ }_{1}^{15}$ which ranges from 0 (no reperfusion) to 3 (full reperfusion in the distribution of the occluded artery) was used to assess recanalization in patients who underwent bridging therapy. A modified $\mathrm{TICl}$ score of $2 \mathrm{~b}$ or 3 (partial reperfusion of more than half of the previously occluded target artery vascular distribution or complete reperfusion, respectively) was considered a successful angiographic outcome. All the cerebral angiographic images were sent to Asan Medical Center for analysis by experts blind to patient clinical and treatment information.
The primary endpoint was a favorable outcome defined by a $\mathrm{mRS}$ of 0 or 1 at 3 months in all the patients randomized. Secondary endpoints included (1) sICH in all randomized patients and (2) favorable outcome (defined by mRS 0 to 1), ordinal distribution of $\mathrm{mRS}$ at 3 months, recanalization rate, and the presence of $\mathrm{sICH}$ in patients who underwent cerebral angiography.

\section{Statistical analysis}

Multivariable logistic regression was used to compare the proportions of patients with favorable outcome (mRS 0 to 1) between the two groups, adjusted for age, sex, initial NIHSS score, time to treatment interval, and baseline variables that were significant at $P<0.1$. Data are reported as odds ratio (OR) and $95 \%$ confidence interval $(\mathrm{Cl})$. Efficacy end points were assessed according to intention-to-treat and per-protocol populations in all Korean patients and in the subset of those who underwent cerebral angiography to consider EVT. In the case of missing data in patients known to be alive, the last observation carried forward approach was used for the outcome assignment; if there was no available data, the worst outcome was assigned. Categorical variables were evaluated with chi-square or Fisher exact tests and continuous variables were compared using a Student t-test. Statistical significance without adjustment for multiple comparisons was set at $\alpha=0.05$. All statistical analyses were performed using SPSS version 21.0 (IBM Co., Armonk, NY, USA). This trial was registered with ClinicalTrials. gov, number NCT01422616.

\section{Results}

From January 24, 2013 through December 3, 2015, there were 361 patients enrolled in the study from 12 Korean centers. Ten patients did not receive alteplase and were thus excluded. Therefore, 351 patients were randomly assigned to an alteplase group (173 to standard-dose, 178 to low-dose) in a modified intention-to-treat population (Figure 1). The per-protocol population included 329 patients (161 in the standard- and 168 in the low-dose group), after excluding 18 patients who were not diagnosed with stroke (10 in the standard- and eight in the low-dose group) and four who were lost to follow-up (two in each the standard- and low-dose groups). Table 1 shows that the baseline demographic and clinical characteristics were well balanced between groups, with the only exception being that there were fewer patients taking aspirin or other antiplatelet agents before the enrollment in the standard-dose group. The results were similar for the per-protocol population.

In the primary endpoint, a favorable outcome occurred in $46 \%$ of patients in both the standard-dose (80/173) and low- 
dose (81/178) groups ( $\mathrm{OR}, 1.03 ; 95 \% \mathrm{Cl}, 0.68$ to $1.57 ; P=0.887)$. As shown in Table 2, the results were similar after adjustment for age, sex, initial NIHSS, time to treatment, and prior use of antiplatelet agents $(\mathrm{OR}, 1.14 ; 95 \% \mathrm{Cl}, 0.72$ to $1.81 ; P=0.582)$. There were no significant differences in mortality or frequency of recurrent ischemic stroke between the groups.

There were 67 patients who underwent cerebral angiography: 33 out of 173 patients who received standard-dose and 34 out of 178 patients who received low-dose alteplase $(P=0.995)$ (Table 1). There were no significant differences in the patients' baseline demographic or clinical characteristics between the standard- and low-dose groups (Table 3). Cerebral angiography revealed that six patients (three from each group) had recanalization after intravenous alteplase. The remaining 61 patients underwent EVT, most frequently with the Solitaire device (Table 3 ). None of the patients received an intra-arterial thrombolytic agent. Between the standard- and low-dose groups, there were no significant differences in the modified $\mathrm{TICl} 2 \mathrm{~b} / 3$ rate $(76 \%$ and $85 \%$, respectively; $P=0.324$ ) or the early neurological improvement rate $(24.2 \%$ and $20.6 \%$, respectively; $P=0.720)$. There was also no significant difference in favorable functional outcome between the standard- and low-dose groups in both the unadjusted $(O \mathrm{R}, 2.51 ; 95 \% \mathrm{Cl}, 0.85$ to $7.43 ; P=0.097)$ and adjusted (OR, 2.39; 95\% Cl, 0.73 to $7.78 ; P=0.149$ ) analyses (Table
2). The results were similar when we investigated the distribution of ordinal mRS scores (Figure 2). There was no heterogeneity of outcome (standard- vs. low-dose) between patients who underwent cerebral angiography and those who did not ( $P$ for interaction $=0.200$ ). When we reanalyzed the data after excluding six patients who had spontaneous recanalization, we found similar results (data not shown).

Of the 49 cases of ICH reported (Table 4), 43 were attributed to alteplase, as determined by responsible physicians. The prevalence of ICH was not significantly different between the treatment groups. Nonetheless, the frequency of sICH with minor neurological deterioration tended to be higher in the standard- than in the low-dose group; however, sICH with major neurological deterioration was rare and the frequency was similar in the two groups. Of the patients who underwent cerebral angiography, there was no significant difference in the frequency of $\mathrm{ICH}$ that was thought to be related to alteplase between the standard- and low-dose groups (15.2\% [5/33] and $14.7 \%$ [5/34], respectively; $P=0.959$ ).

\section{Discussion}

Although the ENCHANTED study has previously reported on the appropriate alteplase dose when patients receive solely intra-

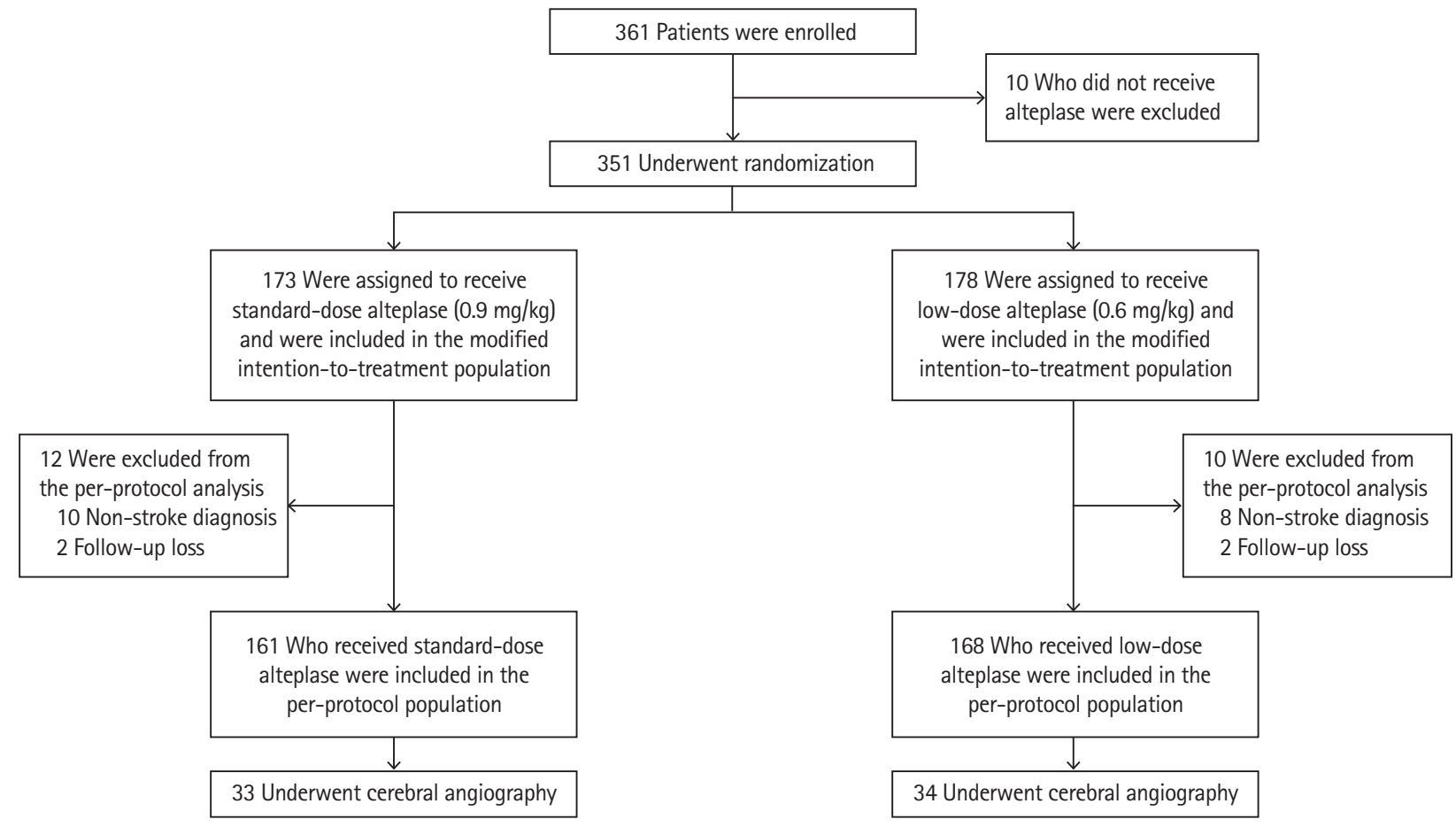

Figure 1. Number of patients who were enrolled, randomly assigned to a study group, and included in the intertion-to-treat and per-protocol populations. The modified intention-to-treat population included patients who were randomized and received alteplase. The per-protocol population included patients who met the criteria of the modified intention-to treat population, had a final diagnosis of ischemic stroke, and completed follow-up. 
Table 1. Baseline demographic and clinical characteristics

\begin{tabular}{|c|c|c|c|}
\hline Variable & Standard-dose alteplase $(n=173)$ & Low-dose alteplase $(n=178)$ & $P$ \\
\hline Male sex & $116(67)$ & $122(69)$ & 0.766 \\
\hline Age (yr) & $66(60-73)$ & $65(57-72)$ & 0.147 \\
\hline Estimated body weight prior to alteplase $(\mathrm{kg})$ & $65 \pm 10$ & $64 \pm 12$ & 0.533 \\
\hline Systolic BP (mmHg) & $145 \pm 23$ & $146 \pm 22$ & 0.625 \\
\hline Diastolic BP $(\mathrm{mmHg})$ & $84 \pm 14$ & $86 \pm 15$ & 0.333 \\
\hline \multicolumn{4}{|l|}{ Risk factors } \\
\hline Previous ischemic stroke & $21(12)$ & $18(10)$ & 0.546 \\
\hline Coronary artery disease & $19(11)$ & $17(10)$ & 0.658 \\
\hline Atrial fibrillation & $28(16)$ & $29(16)$ & 0.978 \\
\hline Hypertension & $95(55)$ & $94(53)$ & 0.693 \\
\hline Diabetes mellitus & $34(20)$ & $40(23)$ & 0.517 \\
\hline Hypercholesterolemia & $29(17)$ & $27(15)$ & 0.683 \\
\hline Current smoker & $55(32)$ & $61(34)$ & 0.622 \\
\hline \multicolumn{4}{|l|}{ Prestroke medication } \\
\hline Warfarin, heparin, or other anticoagulant & $7(4)$ & $9(5)$ & 0.651 \\
\hline Aspirin/other antiplatelet agent & $37(22)$ & $57(32)$ & 0.026 \\
\hline Time to treatment initiation (min) & $115(84-165)$ & $113(88-171)$ & 0.492 \\
\hline \multicolumn{4}{|l|}{ Final diagnosis } \\
\hline Definite ischemic stroke & 161 (95) & $168(96)$ & 0.568 \\
\hline Subtype by TOAST classification & & & 0.693 \\
\hline Large artery disease & $56(35)$ & $65(39)$ & \\
\hline Small vessel disease & $26(16)$ & $26(16)$ & \\
\hline Cardioembolism & $51(32)$ & $50(30)$ & \\
\hline Other & $2(1)$ & $3(2)$ & \\
\hline Undetermined & $26(15)$ & $24(14)$ & \\
\hline NIHSS score at randomization & $8(5-12)$ & $7(4-13)$ & 0.868 \\
\hline Cerebral angiography & $33(19)$ & $34(19)$ & 0.995 \\
\hline
\end{tabular}

Values are presented as number (\%), median (interquartile range), or mean \pm standard deviation.

BP, blood pressure; TOAST, Trial of Org 10172 in Acute Stroke Treatment; NIHSS, National Institutes of Health Stroke Scale.

Table 2. Functional outcome at 3 months

\begin{tabular}{lcccccc}
\hline & $\begin{array}{c}\text { Standard-dose } \\
\text { alteplase favor- } \\
\text { able outcome }\end{array}$ & $\begin{array}{c}\text { Low-dose } \\
\text { alteplase favor- } \\
\text { able outcome }\end{array}$ & OR (95\% Cl) & $P$ & aOR $(95 \% \mathrm{Cl})$ & $P$ \\
\hline All patients & $80 / 173(46)$ & $81 / 178(46)$ & $1.03(0.68-1.57)$ & 0.887 & $1.14(0.72-1.81)$ & 0.582 \\
Patients that undergo cerebral angiography & $13 / 33(39)$ & $7 / 34(21)$ & $2.51(0.85-7.43)$ & 0.097 & $2.39(0.73-7.78)$ & 0.149 \\
$\begin{array}{l}\text { Patients that did not undergo cerebral } \\
\text { angiography }\end{array}$ & $60 / 128(47)$ & $67 / 134(50)$ & $0.88(0.54-1.43)$ & 0.613 & $1.12(0.66-1.91)$ & 0.681 \\
\hline
\end{tabular}

Values are presented as number/total (\%) unless otherwise indicated.

$\mathrm{OR}$, odds ratio; $\mathrm{Cl}$, confidence interval; $\mathrm{aOR}$, adjusted odds ratio.

*Favorable outcome is defined as scores of 1 or less on the modified Rankin Scale; ${ }^{+}$Adjusted for age, sex, initial National Institutes of Health Stroke Scale score, aspirin/other antiplatelet agent, and time to treatment.

venous therapy, this is the first randomized study of alteplase dose in centers where bridging therapy is actively performed. Overall, the results are consistent with those of the main ENCHANTED study. ${ }^{1}$ There was no significant difference in func- tional outcome between the standard- and low-dose alteplase groups (Table 2).

We found that standard-dose alteplase did not substantially reduce the number of patients requiring EVT, nor did it increase 
Table 3. Baseline characteristics of the cerebral angiography subgroup

\begin{tabular}{|c|c|c|c|}
\hline Variable & Standard-dose alteplase $(n=33)$ & Low-dose alteplase $(n=34)$ & $P$ \\
\hline Male sex & $18(53)$ & $22(65)$ & 0.397 \\
\hline Age (yr) & $66(58-74)$ & $69(59-75)$ & 0.705 \\
\hline Involved vessel & & & 0.918 \\
\hline M1 segment of MCA & $14(45)$ & $16(50)$ & \\
\hline M2 segment of MCA & $5(16)$ & $4(13)$ & \\
\hline Internal carotid artery & $9(29)$ & $8(25)$ & \\
\hline Posterior cerebral artery & $1(3)$ & $2(6)$ & \\
\hline Basilar artery & $2(7)$ & $2(6)$ & \\
\hline Mechanical procedure or device & $27(82)$ & 30 (88) & 0.461 \\
\hline Solitaire & $20(74)$ & $19(63)$ & \\
\hline Penumbra & $6(22)$ & $6(20)$ & \\
\hline Angioplasty & 0 & $1(3)$ & \\
\hline Other & $2(7)$ & $4(13)$ & \\
\hline Successful recanalization* & $25(76)$ & $29(85)$ & 0.324 \\
\hline Recanalization after alteplase & 3 & 3 & \\
\hline Recanalization after intervention & 22 & 29 & \\
\hline TOAST classification & & & 0.726 \\
\hline Large artery disease & $12(36)$ & $15(44)$ & \\
\hline Small vessel disease & 0 & 0 & \\
\hline Cardioembolism & $18(55)$ & $15(44)$ & \\
\hline Other determined & 0 & 0 & \\
\hline Undetermined & $3(9.1)$ & $4(11.8)$ & \\
\hline NIHSS score on admission & $13(9-16)$ & $13(8-19)$ & 0.353 \\
\hline NIHSS score at day 3 & $7(4-16)$ & $8(5-16)$ & 0.913 \\
\hline Early neurologic improvement ${ }^{+}$ & $8(24)$ & $7(21)$ & 0.720 \\
\hline
\end{tabular}

Values are presented as number (\%) or median (interquartile range).

MCA, middle cerebral artery; TOAST, Trial of Org 10172 in Acute Stroke Treatment; NIHSS, National Institutes of Health Stroke Scale.

*Successful recanalization is defined as modified thrombolysis in cerebral infarction grade $2 b$ or 3 , indicating partial reperfusion of more than half of the previously occluded target artery vascular distribution, or complete reperfusion; 'Early neurologic improvement defined as a reduction of $\geq 8$ points on the NIHSS or a score of 0 or 1 at 3 days.

the rate of recanalization in patients who underwent EVT. It is recognized that standard-dose alteplase does not effectively recanalize large cerebral artery occlusions, with frequencies ranging from $6 \%$ for distal internal carotid artery occlusions to $30 \%$ for proximal middle cerebral artery occlusions. ${ }^{16}$ As our study did not have a control group, i.e., a group of patients who did not receive alteplase, our results do not imply that intravenous alteplase is ineffective at recanalizing an occluded artery, but instead show that standard-dose was not substantially superior to low-dose alteplase in this regard.

It should be noted that our finding of similar angiographic recanalization rates on the post-alteplase angiogram does not necessarily indicate a comparable effect of standard- and lowdose alteplase on arterial recanalization. Because cerebral angiography was usually performed shortly after intravenous al- teplase infusion, it may not have detected delayed recanalization resulting from the infusion. However, because there was also no evidence of a higher recanalization rate even after EVT in the standard-dose group, standard-dose alteplase does not appear to have a superior recanalizing effect than low-dose alteplase even when it is combined with EVT.

When investigating hemorrhagic complications, the main ENCHANTED trial found that low-dose alteplase was associated with a significantly lower frequency of sICH than standarddose alteplase. ${ }^{1}$ Although the rate of sICH did not significantly differ between the two groups in the current study, there tended to be more sICHs in the standard-dose group. Nevertheless, $\mathrm{sICHs}$ were rarely associated with major neurological deterioration and there also was no difference in the $\mathrm{ICH}$ rates according to a different dose of alteplase in patients who under- 
Table 4. Frequency of intracerebral hemorrhage

\begin{tabular}{|c|c|c|c|c|}
\hline \multirow[b]{2}{*}{ Symptomatic ICHs outcome } & \multicolumn{3}{|c|}{ Intention-to-treat population } & \multirow[b]{2}{*}{$P$} \\
\hline & $\begin{array}{l}\text { Standard-dose alteplase } \\
\qquad(n=173)\end{array}$ & $\begin{array}{l}\text { Low-dose alteplase } \\
\qquad(n=178)\end{array}$ & $\mathrm{OR}(95 \% \mathrm{Cl})$ & \\
\hline Total reported ICH & $27(16)$ & $22(12)$ & $1.31(0.75-2.41)$ & 0.380 \\
\hline Any hemorrhage at 24 hours & $22(13)$ & $21(12)$ & $1.11(0.59-2.11)$ & 0.745 \\
\hline ICH presumed to be related to alteplase ${ }^{*}$ & $23(13)$ & $20(11)$ & $1.21(0.64-2.30)$ & 0.556 \\
\hline Symptomatic $\mathrm{ICH}^{+}$ & $14(8)$ & $6(3)$ & $2.53(0.95-6.73)$ & 0.056 \\
\hline Minor neurological deterioration & $8(5)$ & $2(1)$ & $4.27(0.89-20.39)$ & 0.059 \\
\hline Major neurological deterioration & $6(4)$ & $4(2)$ & $1.56(0.43-5.64)$ & 0.538 \\
\hline Resolution & & & & 0.757 \\
\hline Resolved completely & $16(70)$ & $15(75)$ & & \\
\hline Sustained lasting damage & $2(9)$ & $1(5)$ & & \\
\hline Resulted in death & $5(22)$ & $4(20)$ & & \\
\hline
\end{tabular}

Values are presented as number (\%).

$\mathrm{OR}$, odds ratio; $\mathrm{Cl}$, confidence interval; ICH, intracerebral hemorrhage.

*According to the opinion of charged physician; ${ }^{+}$Symptomatic ICH is defined as ICH with $\geq 1$-point deterioration on the National Institute of Health Stroke Scale (NIHSS) as confirmed by brain imaging or death within 36 hours from baseline. Minor neurological deterioration is defined as 1-3 points deterioration on the NIHSS, major neurological deterioration is defined as $\geq 4$ points decline in NIHSS score over 72 hours

\section{Distribution of $\mathrm{mRS}$ scores at 3 months}

Modified intention-to-treat population

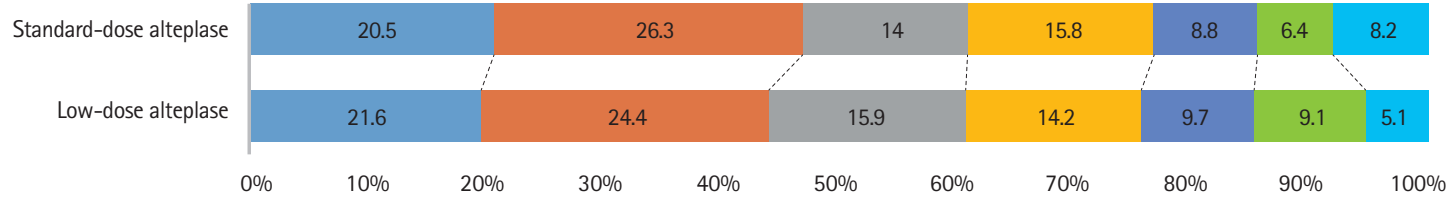

Per-protocol population

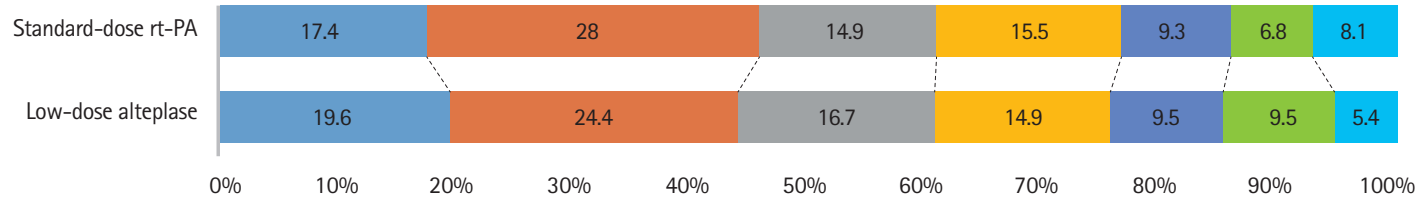

Cerebral angiography subgroup

\begin{tabular}{|c|c|c|c|c|c|c|c|c|c|c|c|}
\hline Standard-dose rt-PA & 6.1 & & 33 & & & 18.2 & 12.1 & & & 15.2 & 6.1 \\
\hline Low-dose alteplase & 5.9 & 14.7 & & 17.6 & & 17.6 & & 23.5 & & 14.7 & 5.9 \\
\hline & $0 \%$ & $10 \%$ & $20 \%$ & $30 \%$ & $40 \%$ & $50 \%$ & $60 \%$ & $70 \%$ & $80 \%$ & $90 \%$ & $100 \%$ \\
\hline
\end{tabular}

Figure 2. Distribution of scores on the modified Rankin Scale (mRS). The distribution of mRS score is shown for the intention-to-treat population, per-protocol population, and cerebral angiography subgroup at the 90-day follow-up. rt-PA, recombinant tissue plasminogen activator.

went bridging therapy. Thus, it seems that alteplase-associated sICHs are not more problematic in our setting than when alteplase intravenous therapy is used alone. However, the low number of enrolled patients and ICH cases precludes any definitive conclusion.
Interestingly, despite similar recanalization rates between the groups, we found a trend towards more favorable functional outcome in the standard-dose as compared to the lowdose alteplase group, at least in patients who underwent cerebral angiography (Table 2 and Figure 2). Although the differ- 
ence was not statistically significant, this result raises an intriguing possibility that standard-dose alteplase may recanalize smaller arteries more effectively; thereby, improving antegrade perfusion or collateral circulation in patients with large artery occlusions. Further studies that enroll a larger number of patients undergoing bridging therapy should be performed to confirm or refute our preliminary observation.

We recognize that our study has several limitations. First, although South Korea performed EVT most actively among the 13 countries involved in the ENCHANTED study, the rate of bridging therapy in the current study was below 20\% partly because the patients were enrolled before the publication of positive EVT trials. Therefore, we suspect that results might be different if similar studies are performed in the future when EVTs are more actively performed. Second, as the patients in this study were all Korean, the generalizability of our findings to current practice in other regions may be questioned. Finally, there were a small number of patients who underwent EVT and thus, the study was not adequately powered to compare the outcome of patients who underwent bridging therapy.

Despite these limitations, our study was the first randomized controlled study that examined the dose of alteplase in the context of bridging therapy. Our data illustrate that the functional outcome was similar between the standard-dose and low-dose alteplase groups despite slightly increased sICHs in the former group, consistent with the findings from a recent meta-analysis. ${ }^{17}$ We also observed a potential superiority of standard-dose alteplase in the functional outcome of patients who underwent EVT. Therefore, we suggest that standard-dose alteplase be maintained as standard care in centers where EVT is actively performed as suggested by a recent guideline ${ }^{18}$ until more definitive data is available from future large, prospective, multinational trials.

\section{Disclosure}

Thompson G. Robinson is a National Institute of Health Research Senior Investigator and reports receiving speaking fees from Bayer and Boehringer Ingelheim and fees for serving on Advisory Panels from Bayer and Daiichi Sankyo. Craig S. Anderson reports receiving fees for serving on Advisory Panels from Astra Zeneca and Medtronic, speaking at seminars for Takeda China and Boehringer Ingelheim, and receiving a research grant from Takeda China. John Chalmers reports research grants and lecture fees from Servier for the ADVANCE trial and post-trial follow-up. Richard I. Lindley reports receiving speaking fees from Boehringer Ingelheim, Covidien, and Pfizer.

\section{Acknowledgments}

This study was funded by the Ministry for Health, Welfare, and Family Affairs of the Republic of Korea (HI14C1985), National Health and Medical Research Council (NHMRC) of Australia, and the Stroke Association of the United Kingdom. These agencies had no role in the design of the study protocol; collection, analysis, or interpretation of trial data; or writing of the manuscript.

\section{References}

1. Anderson CS, Robinson T, Lindley Rl, Arima H, Lavados PM, Lee $\mathrm{TH}$, et al. Low-dose versus standard-dose intravenous alteplase in acute ischemic stroke. N Engl J Med 2016;374:2313-2323.

2. Berkhemer OA, Fransen PS, Beumer D, van den Berg LA, Lingsma HF, Yoo AJ, et al. A randomized trial of intraarterial treatment for acute ischemic stroke. N Engl J Med 2015;372:11-20.

3. Jovin TG, Chamorro A, Cobo $E$, de Miquel MA, Molina CA, Rovira $A$, et al. Thrombectomy within 8 hours after symptom onset in ischemic stroke. N Eng/J Med 2015;372:2296-2306.

4. Goyal M, Demchuk AM, Menon BK, Eesa M, Rempel JL, Thornton $J$, et al. Randomized assessment of rapid endovascular treatment of ischemic stroke. NEngl J Med 2015;372:1019-1030.

5. Campbell BC, Mitchell PJ, Kleinig TJ, Dewey HM, Churilov L, Yassi $\mathrm{N}$, et al. Endovascular therapy for ischemic stroke with perfusion-imaging selection. N Engl J Med 2015;372:1009-1018.

6. Saver JL, Goyal M, Bonafe A, Diener HC, Levy El, Pereira VM, et al. Stent-retriever thrombectomy after intravenous t-PA vs. t-PA alone in stroke. N Eng J Med 2015;372:2285-2295.

7. Hong KS, Ko SB, Lee JS, Yu KH, Rha JH. Endovascular recanalization therapy in acute ischemic stroke: updated meta-analysis of randomized controlled trials. J Stroke 2015;17:268-281.

8. Huang $Y$, Sharma VK, Robinson T, Lindley RI, Chen $X$, Kim JS, et al. Rationale, design, and progress of the enhanced control of hypertension and thrombolysis stroke study (ENCHANTED) trial: an international multicenter $2 \times 2$ quasi-factorial randomized controlled trial of low- vs. standard-dose rt-PA and early intensive vs. guideline-recommended blood pressure lowering in patients with acute ischaemic stroke eligible for thrombolysis treatment. Int J Stroke 2015;10:778-788.

9. Anderson CS, Woodward M, Arima $H_{1}$ Chen $X$, Lindley RI, Wang $X$, et al. Statistical analysis plan for evaluating low- vs. standard-dose alteplase in the enhanced control of hypertension and thrombolysis stroke study (ENCHANTED). Int J Stroke 2015;10:1313-1315.

10. Quinn TJ, Dawson J, Walters MR, Lees KR. Functional outcome measures in contemporary stroke trials. Int J Stroke 2009;4:200-205. 
11. Lyden P, Brott T, Tilley B, Welch KM, Mascha EJ, Levine $S$, et al. Improved reliability of the NIH stroke scale using video training. NINDS TPA Stroke Study Group. Stroke 1994;25:2220-2226.

12. National Institute of Neurological Disorders and Stroke rt-PA Stroke Study Group. Tissue plasminogen activator for acute ischemic stroke. N Eng J Med 1995;333:1581-1587.

13. Hacke W, Kaste M, Bluhmki E, Brozman M, Dávalos A, Guidetti $D$, et al. Thrombolysis with alteplase 3 to 4.5 hours after acute ischemic stroke. N Engl J Med 2008;359:1317-1329.

14. Wahlgren N, Ahmed N, Dávalos A, Ford GA, Grond M, Hacke $W$, et al. Thrombolysis with alteplase for acute ischaemic stroke in the safe implementation of thrombolysis in StrokeMonitoring Study (SITS-MOST): an observational study. Lancet 2007;369:275-282.

15. Tomsick T, Broderick J, Carrozella J, Khatri P, Hill M, Palesch $Y$, et al. Revascularization results in the interventional Man- agement Of Stroke II Trial. AJNR AJNR Am J Neuroradiol 2008;29:582-587.

16. Saqqur M, Uchino $K$, Demchuk AM, Molina CA, Garami Z, Calleja $S$, et al. Site of arterial occlusion identified by transcranial Doppler predicts the response to intravenous thrombolysis for stroke. Stroke 2007;38:948-954.

17. Georgiadis $A L$, Memon $M Z$, Shah $Q A$, Vazquez G, Suri MF, Lakshminarayan $K_{1}$ et al. Comparison of partial $(.6 \mathrm{mg} / \mathrm{kg}$ ) versus full-dose $(.9 \mathrm{mg} / \mathrm{kg})$ intravenous recombinant tissue plasminogen activator followed by endovascular treatment for acute ischemic stroke: a meta-analysis. J Neuroimaging 2011;21:113-120.

18. Hong KS, Ko SB, Yu KH, Jung C, Park SO, Kim BM, et al. Update of the Korean clinical practice guidelines for endovascular recanalization therapy in patients with acute ischemic stroke. J Stroke 2016;18:102-113. 